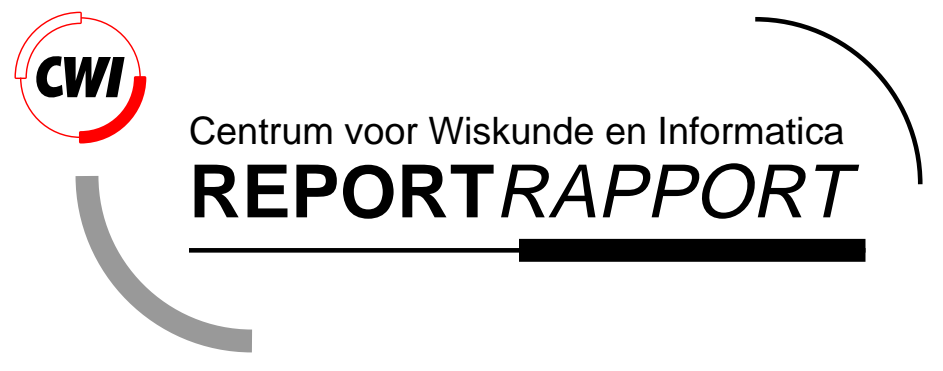

Disturbance decoupling and robustness of stability

U. Baser, M.K.K. Cevik, J.M. Schumacher

Modelling, Analysis and Simulation (MAS)

MAS-R9827 December 1998 
Report MAS-R9827

ISSN 1386-3703

CWI

P.O. Box 94079

1090 GB Amsterdam

The Netherlands

CWI is the National Research Institute for Mathematics and Computer Science. CWI is part of the Stichting Mathematisch Centrum (SMC), the Dutch foundation for promotion of mathematics and computer science and their applications.

SMC is sponsored by the Netherlands Organization for Scientific Research (NWO). CWI is a member of ERCIM, the European Research Consortium for Informatics and Mathematics.

Copyright (C) Stichting Mathematisch Centrum P.O. Box 94079, 1090 GB Amsterdam (NL) Kruislaan 413, 1098 SJ Amsterdam (NL) Telephone +3120 5929333 Telefax +31205924199 


\title{
Disturbance Decoupling and Robustness of Stability
}

\author{
U. Bașer \\ Department of Mathematics, Istanbul Technical University \\ 80626 Maslak, Istanbul, Turkey \\ Baser@sariyer.cc.itu.edu.tr \\ M.K.K. Cevik \\ Electrical and Electronics Engineering Faculty \\ Istanbul Technical University \\ 80626 Maslak, Istanbul, Turkey \\ eecevik@titan.ehb.itu.edu.tr \\ J.M. Schumacher \\ CWI, P.O. Box 94079, 1090 GB Amsterdam, The Netherlands, and \\ Tilburg University, CentER and Department of Economics, \\ P.O. Box 90153, 5000 LE Tilburg, The Netherlands \\ Hans.Schumacher@cwi.nl
}

\begin{abstract}
In this paper we investigate the interaction of disturbance decoupling requirement with the robustness of closed loop stability in the sense of normalized coprime factor perturbations. The disturbance decoupling and the robust stabilization requirements are expressed in terms of the subspace valued functions associated to plant and controller and an upper bound on robustness of stability imposed by the disturbance decoupling requirement is derived. This is then compared with the actual robustness of stability that can be achieved by a solution of the disturbance decoupling problem for systems having left invertible transfer matrix from the control inputs to the controlled outputs.
\end{abstract}

1991 Mathematics Subject Classification: 93B35, 93B50, 93 C05.

Keywords and Phrases: linear systems, stability, robustness, disturbance decoupling.

Note: Work carried out in part under project MAS2.4 "Discontinuous dynamical systems".

\section{Introduction}

The problem of designing a feedback controller to make the output of a system insensitive to disturbances acting at the input is called the disturbance decoupling problem. The original solution of this problem assuming that the whole state vector is accessible for measurement is one of the problems that motivated the geometric approach to system theory discovered by Basile, Marro, Wonham, and Morse [21, 1]. Later, the problem has been solved by dynamic measurement feedback and under the additional condition of closed loop stability. See for instance $[16,10,20,13]$ and the references therein. These studies basically concentrate on the solvability of the problem for a given set of system parameters. However in applications the system description is seldom precisely known and the problem should be solved by taking the uncertainties into account. This requires a model for the uncertainties to be developed. Various approaches have been suggested in the literature. Here we consider the model based on the perturbation of the normalized coprime factors of the plant. The problem of robustly stabilizing a plant with respect to this perturbation class has been solved by Glover and MacFarlane [8].

The purpose of this paper is to investigate the interaction between the disturbance decoupling requirement and the requirement of robust closed loop stability and to explore the role of finite 
dimensional geometry to explain this interaction. For this purpose the disturbance decoupling and robust stability requirements are expressed in terms of subspace valued functions associated to plant and controller. Finite dimensional geometry then readily leads to an upper bound on the achievable robustness of stability. For systems having left invertible transfer matrices from the control inputs to the controlled outputs the maximum robustness of stability that can be achieved by a solution of the disturbance decoupling problem is determined and compared with the upper bound obtained by the pointwise geometrical analysis.

The idea of associating to a finite dimensional linear time invariant system with $m$ inputs and $p$ outputs, a function from the complex plane to subspaces of $(m+p)$ - dimensional space has been introduced by Martin and Hermann [12]. In [2,3] it has been shown that subspace valued functions can be used to describe the interaction between the design objectives of regulation and robust closed loop stability. The treatment of the problem in the present paper follows these papers. However in contrast with the main result of [2] it is shown that in the case of disturbance decoupling problem the upper bound on the robustness measure derived by the pointwise geometrical analysis is not sharp and in general gives optimistic results.

The paper is organized as follows. In the next section subspace valued functions are introduced starting from a state space description of the plant and controller and the problem studied in the paper is precisely defined. In section 3 the disturbance decoupling problem with internal stability is formulated in terms of subspace valued functions and an upper bound on the robustness of stability is derived. For a class of systems satisfying the left invertibility condition mentioned above the general solution of the disturbance decoupling problem with stability is parametrized in section 4 and the actual robustness of stability that can be attained by a solution of the disturbance decoupling problem is determined by an $H_{\infty}$ optimization procedure. The upper bound derived by the geometric analysis of section 3, the overall bound on robustness of stability and the actual robustness of stability are computed and compared with each other on an example in section 5 . The paper ends with section 6 containing some conclusions and suggestions for further study.

The notation follows standard linear systems literature, see $[21,5]$ for instance. Some of the frequently used symbols are listed below for convenience.

\section{List of symbols}

DDIS Disturbance decoupling problem with internal stability.

DDRS Disturbance decoupling problem with robust stability.

$\mathcal{Z}, \mathcal{Y}, \mathcal{D}, \mathcal{U} \quad$ Finite dimensional spaces of controlled output, measured output, distur-

bance input and control input variables respectively.

$\mathcal{W} \quad \mathcal{Z} \times \mathcal{Y} \times \mathcal{D} \times \mathcal{U}$ external variable space.

$\mathcal{K} \quad\{0\} \times \mathcal{Y} \times \mathcal{D} \times \mathcal{U}$.

$\mathcal{Q} \quad \mathcal{Z} \times \mathcal{Y} \times\{0\} \times \mathcal{U}$.

$\mathcal{N} \quad \mathcal{Z} \times\{0\} \times \mathcal{D} \times\{0\}$.

$\Pi: \mathcal{W} \mapsto \mathcal{Y} \times \mathcal{U} \quad$ Projection onto $\mathcal{Y} \times \mathcal{U}$ along $\mathcal{N}$.

$\mathbb{C}^{+} \quad\{s \in \mathrm{C} \mid \operatorname{Re}(s) \geq 0\} \cup\{\infty\}$ closed right half plane.

$R H_{\infty} \quad$ Real rational functions that are analytic in $\mathbb{C}^{+}$.

2. Problem Formulation AND PReliminaries

Consider the finite-dimensional linear time-invariant system described by

$$
\begin{aligned}
\dot{x}(t) & =A x(t)+B u(t)+E d(t) \\
y(t) & =C x(t) \\
z(t) & =D x(t) .
\end{aligned}
$$

The equations represent a plant with state $x \in \mathcal{X}$, control input $u \in \mathcal{U}$, measurements $y \in \mathcal{Y}$, and controlled outputs $z \in \mathcal{Z}$ subject to the disturbances $d \in \mathcal{D}$. Here $\mathcal{X}, \mathcal{U}, \mathcal{D}, \mathcal{Y}$ and $\mathcal{Z}$ are vector spaces 
of dimensions $n, m, r, p$ and $\ell$ respectively. It is assumed that the pair $(A, B)$ is stabilizable and the pair $(C, A)$ is detectable.

The system is controlled by a feedback compensator defined by

$$
\begin{aligned}
\dot{x}_{c}(t) & =F x_{c}(t)+G y(t) \\
u(t) & =H x_{c}(t)+J y(t) .
\end{aligned}
$$

We also assume that the pair $(F, G)$ is stabilizable and the pair $(H, F)$ is detectable. The closed loop system is given by

$$
\begin{aligned}
\dot{x}_{e}(t) & =A_{e} x_{e}(t)+E_{e} d(t) \\
z(t) & =D_{e} x_{e}(t)
\end{aligned}
$$

where

$$
x_{e}(t)=\left[\begin{array}{c}
x(t) \\
x_{c}(t)
\end{array}\right], \quad A_{e}=\left[\begin{array}{cc}
A+B J C & B H \\
G C & F
\end{array}\right], \quad E_{e}=\left[\begin{array}{c}
E \\
0
\end{array}\right], \quad D_{e}=\left[\begin{array}{cc}
D & 0
\end{array}\right] .
$$

The closed loop system is internally stable if the eigenvalues of $A_{e}$ have negative real parts and disturbance decoupled if the transfer function from the disturbance input $d(t)$ to the controlled output $z(t)$ is identically zero. The controller is a solution of the disturbance decoupling problem with internal stability (DDIS) if the closed loop system is disturbance decoupled and internally stable. Note that the closed loop transfer function can be written as

$$
G_{\mathrm{cl}}(s)=G_{11}(s)+G_{12}(s) K(s)\left(I-G_{22}(s) K(s)\right)^{-1} G_{21}(s)
$$

where $\left[\begin{array}{ll}G_{11}(s) & G_{12}(s) \\ G_{21}(s) & G_{22}(s)\end{array}\right]=\left[\begin{array}{l}D \\ C\end{array}\right](s I-A)^{-1}\left[\begin{array}{ll}E & B\end{array}\right]$ is the transfer function of the system (2.1-2.3) and $K(s)=H(s I-F)^{-1} G+J$ is the transfer function of the controller. Under the stabilizability and detectability assumptions we have made the internal stability of the closed loop system is equivalent to $[18$, Lemma 5.1.17]

$$
\left[\begin{array}{cc}
I & G_{22}(s) \\
K(s) & I
\end{array}\right]^{-1} \in R H_{\infty}^{(m+p) \times(m+p)} .
$$

Thus the problem DDIS amounts to finding a proper, rational function $K(s)$ that satisfies $(2.10)$ and (2.9) with $G_{\mathrm{cl}}(s)=0$.

In the sequel, we need a re-formulation of the disturbance decoupling and internal stability requirements in terms of the subspace valued functions defined below. Consider the equations associated to the plant (2.1-2.3) and the controller (2.4-2.5).

$$
\begin{aligned}
& {\left[\begin{array}{ccccc}
s I-A & 0 & 0 & -E & -B \\
C & 0 & -I & 0 & 0 \\
D & -I & 0 & 0 & 0
\end{array}\right]\left[\begin{array}{l}
x \\
z \\
y \\
d \\
u
\end{array}\right]=0} \\
& {\left[\begin{array}{ccc}
s I-F & -G & 0 \\
H & J & -I
\end{array}\right]\left[\begin{array}{c}
x_{c} \\
y \\
u
\end{array}\right]=0}
\end{aligned}
$$

Write $\mathcal{W}=\mathcal{Z} \times \mathcal{Y} \times \mathcal{D} \times \mathcal{U}$ for the external variable space with $\operatorname{dim} \mathcal{W}=q$ and let $w=\operatorname{col}(z y d u)$ denote the $q$ - dimensional vector of external variables. The subspace valued function associated to the plant is defined as

$$
\mathcal{P}(s)=\{w \in \mathcal{W} \mid \exists x \text { s.t. (2.11) holds }\}, \mathcal{P}(\infty)=\{0\} \times\{0\} \times \mathcal{D} \times \mathcal{U}
$$


Similarly, the subspace valued function associated to the controller is defined by

$$
\mathcal{C}(s)=\left\{w \in \mathcal{W} \mid \exists x_{c} \text { s.t. (2.12) holds }\right\}, \mathcal{C}(\infty)=\operatorname{ker}[0 J 0-I] .
$$

The functions $\mathcal{P}(s)$ and $\mathcal{C}(s)$ are considered as functions from the closed right half plane $\mathbb{C}^{+}$to the Grassmannian manifold of subspaces of $\mathcal{W}$. From the stabilizability and detectability assumptions it follows that [4, Lemma 2.3]

$$
\operatorname{dim} \mathcal{P}(s)=m+r \text { and } \operatorname{dim} \mathcal{C}(s)=p+r+\ell \text { for all } s \in \mathbb{C}^{+} .
$$

Subspace valued functions may be alternatively characterized as image or kernel representations corresponding to coprime factorizations of the transfer matrices. In fact Martin and Hermann used polynomial coprime factorizations in their original paper [12]. Since we consider stabilizable and detectable systems it is more convenient to use factorizations over $R H_{\infty}$. The equivalence of image and kernel representations to the above definitions is shown in [4, Lemma 2.4]. Throughout the paper the subspaces $\mathcal{Q}, \mathcal{K}, \mathcal{N}$ are defined by

$$
\begin{aligned}
& \mathcal{Q}=\mathcal{Z} \times \mathcal{Y} \times\{0\} \times \mathcal{U} \\
& \mathcal{K}=\{0\} \times \mathcal{Y} \times \mathcal{D} \times \mathcal{U} \\
& \mathcal{N}=\mathcal{Z} \times\{0\} \times \mathcal{D} \times\{0\}
\end{aligned}
$$

and $\Pi: \mathcal{W} \mapsto \mathcal{Y} \times \mathcal{U}$ denotes the natural projection onto $\mathcal{Y} \times \mathcal{U}$ along $\mathcal{N}$. Note that $\mathcal{C}(s)$ satisfies

$$
\mathcal{N} \subset \mathcal{C}(s) \text { for all } s \in \mathbb{C}^{+}
$$

and

$$
\mathcal{C}(\infty) \oplus\{0\} \times\{0\} \times\{0\} \times \mathcal{U}=\mathcal{W} .
$$

The relation (2.19) holds because the controller gives a relation between $y$ and $u$ even though we defined $\mathcal{C}(s)$ with external variable space $\mathcal{W}$ rather than $\mathcal{Y} \times \mathcal{U}$. Conversely, a subspace valued function $\mathcal{C}(s)$ given by image or kernel representation can be realized as a proper controller with inputs $y$ and outputs $u$ if $(2.19,2.20)$ are satisfied [6]. Therefore we will call $(2.19,2.20)$ realizability conditions. The next lemma expresses the internal stability of the closed loop system in terms of the subspace values functions defined above. This is essentially the same as [3, Lemma 2.5] but an alternative proof is given here for completeness.

LEMma 2.1 Consider the system (2.1-2.3) and the controller (2.4, 2.5). Assume that the pairs (A,B) and $(F, G)$ are stabilizable and the pairs $(C, A)$ and $(H, F)$ are detectable. Under these assumptions the closed loop system (2.6, 2.7) is internally stable if and only if

$$
\mathcal{C}(s) \oplus \mathcal{P}(s) \cap \mathcal{Q}=\mathcal{W} \text { for all } s \text { with } \operatorname{Re}(s) \geq 0 .
$$

Proof For $d(t)=0$ assume that the solution of the closed loop system is of the form $x(t)=$ $x_{0} e^{\lambda t}, x_{c}(t)=x_{c 0} e^{\lambda t}$ and similarly for $z(t), y(t), u(t)$. Substituting the assumed solutions in (2.1-2.3) and $(2.4,2.5)$ and equating the coefficients of $e^{\lambda t}$ results in

$$
\left[\begin{array}{cccc}
\lambda I-A & 0 & 0 & -B \\
C & 0 & -I & 0 \\
D & -I & 0 & 0
\end{array}\right]\left[\begin{array}{c}
x_{0} \\
z_{0} \\
y_{0} \\
u_{0}
\end{array}\right]=0, \quad\left[\begin{array}{ccc}
\lambda I-F & -G & 0 \\
H & J & -I
\end{array}\right]\left[\begin{array}{c}
x_{c 0} \\
y_{0} \\
u_{0}
\end{array}\right]=0
$$

The closed loop system is stable if and only if for $\operatorname{Re}(\lambda) \geq 0$ the equations $(2.22)$ have only the trivial solution $x_{0}=0, x_{c 0}=0, z_{0}=0, y_{0}=0, u_{0}=0$ in common which is equivalent to $\mathcal{C}(\lambda) \cap \mathcal{P}(\lambda) \cap \mathcal{Q}=\{0\}$. Note that

$$
\mathcal{P}(s) \cap \mathcal{Q}=\left[\begin{array}{ll}
D & 0 \\
C & 0 \\
0 & 0 \\
0 & I
\end{array}\right] \operatorname{ker}[s I-A-B]
$$


and as a consequence of the detectability of the pair $(C, A)$ and stabilizability of the pair $(A, B)$ we have $\operatorname{dim}(\mathcal{P}(s) \cap \mathcal{Q})=m$ for $\operatorname{Re}(s) \geq 0$. Thus $\operatorname{dim}(\mathcal{P}(s) \cap \mathcal{Q}+\mathcal{C}(s))=\operatorname{dim}(\mathcal{P}(s) \cap \mathcal{Q})+\operatorname{dim} \mathcal{C}(s)=\operatorname{dim} \mathcal{W}$ for $\operatorname{Re}(s) \geq 0$ which establishes $(2.21)$.

It is well known that the controller stabilizes the system if and only if it stabilizes the subsystem with transfer function $G_{22}(s)$ [5, Thm. 4.3.2]. This statement is translated into the language of subspace valued functions by projecting the subspaces in $(2.21)$ to $\mathcal{Y} \times \mathcal{U}$.

LEMMA 2.2 Under the assumptions of Lemma 2.1 the closed loop system is stable if and only if

$$
\Pi \mathcal{C}(s) \oplus \Pi(\mathcal{P}(s) \cap \mathcal{Q})=\mathcal{Y} \times \mathcal{U} \quad \text { for all } s \text { with } \operatorname{Re}(s) \geq 0
$$

Proof Since $\mathcal{N}=\operatorname{ker} \Pi \subset \mathcal{C}(s)$ (2.21) implies (2.24). Conversely (2.24) implies (2.21) under the additional condition $\mathcal{P}(s) \cap \mathcal{N} \cap \mathcal{Q}=\{0\}$ for $\operatorname{Re}(s) \geq 0$ which follows from the detectability of the pair $(C, A)$.

The robustness of closed loop stability is measured by the minimal angle between the complementary subspaces in (2.24). The minimal angle between two subspaces $\mathcal{Y}$ and $\mathcal{Z}$ of a unitary space $\mathcal{X}$ is defined as (see for instance [9, p. 339])

$$
\sin \phi(\mathcal{Y}, \mathcal{Z})=\min \{\|y-z\| \mid y \in \mathcal{Y}, \quad z \in \mathcal{Z},\|y\|=1\}, \quad 0 \leq \phi \leq \frac{1}{2} \pi
$$

If $\mathcal{Y}, \mathcal{Z}$ are complementary subspaces another formula for the minimal angle is given by [9, p. 339]

$$
\sin \phi(\mathcal{Y}, \mathcal{Z})=\left\|\Pi_{\mathcal{Y}}^{\mathcal{Z}}\right\|^{-1}
$$

where $\Pi_{\mathcal{Y}}^{\mathcal{Z}}$ is the projection on $\mathcal{Z}$ along $\mathcal{Y}$. In this case $(2.25)$ gives the shortest distance of $\mathcal{Y}$ (in the sense of gap metric) to subspaces that are not complementary to $\mathcal{Z}$ [17]. As a measure of robustness of stability we shall therefore take

$$
\min _{s \in \mathbb{C}+} \sin \phi(\Pi(\mathcal{P}(s) \cap \mathcal{Q}), \Pi \mathcal{C}(s)) .
$$

When the closed loop system is stable, using the characterization $(2.26),(2.27)$ can be written as

$$
\left\|\left[\begin{array}{c}
I \\
K(s)
\end{array}\right]\left(I-G_{22}(s) K(s)\right)^{-1}\left[\begin{array}{ll}
I & -G_{22}(s)
\end{array}\right]\right\|_{\infty}^{-1}
$$

and coincides with the robustness measure used in the works $[19,8,7,14]$. We now define the problem considered in this paper.

Problem (Disturbance decoupling problem with robust stability margin $\gamma: \operatorname{DDRS}(\gamma)$ )

Given the plant (2.1-2.3) and $\gamma$ with $0<\gamma<1$, find a compensator of the form (2.4-2.5) such that the closed loop system is disturbance decoupled and the robust stability condition

$$
\min _{s \in \mathbb{C}+} \sin \phi(\Pi(\mathcal{P}(s) \cap \mathcal{Q}), \Pi \mathcal{C}(s)) \geq \gamma
$$

is satisfied.

The solution of the disturbance decoupling problem by dynamic measurement feedback has been given in $[1$, Ch.5] while the problem of finding a controller that satisfies the robust stabilization condition (2.28) has been solved in [8]. The solvability of these problems are clearly necessary for the solvability of $\operatorname{DDRS}(\gamma)$. In this paper we derive an upper bound on the robustness measure (2.27) under the constraint that the controller satisfies the disturbance decoupling requirement. This provides another necessary condition for the solution of the problem defined above. We then compute the maximal value of $\gamma$ for which $\operatorname{DDRS}(\gamma)$ is solvable under an additional assumption and compare with the upper bound derived. 
3. AN UPPER BOUND ON ROBUSTNESS OF STABILITY

We first give conditions on the subspace valued function of the controller for the disturbance decoupling and internal stability requirements to be satisfied; then these conditions are used to derive an upper bound on the achievable robusness of stability.

THEOREM 3.1 A controller with subspace valued function $\mathcal{C}(s)$ is a solution of the disturbance decoupling problem with internal stability if and only if for all $s$ in $\mathbb{C}^{+}$

$$
\begin{aligned}
& \mathcal{C}(s) \oplus \mathcal{P}(s) \cap \mathcal{Q}=\mathcal{W} \\
& \mathcal{P}(s) \cap \mathcal{C}(s) \subset \mathcal{K} \\
& \mathcal{N} \subset \mathcal{C}(s) .
\end{aligned}
$$

Proof The conditions (3.3) and (3.1) at $s=\infty$ are the realizability conditions of the controller as a proper system with inputs $y$ and outputs $u$. The condition (3.1) is equivalent to the internal stability of the closed loop system. Under this condition, the solution of the equations (2.11) and (2.12) for $\operatorname{Re}(s) \geq 0$ satisfies $z=G_{\mathrm{cl}}(s) d$ where $G_{\mathrm{cl}}(s)$ is the closed loop transfer function. Hence the closed loop system is disturbance decoupled if and only if the solution of these equations satisfies $z=0$. This is equivalent to (3.2).

For given $s \in \mathbb{C}^{+}$we now denote the values of $\mathcal{P}(s), \mathcal{C}(s)$ by $\mathcal{P}$ and $\mathcal{C}$ and consider the purely geometric problem of finding the maximum of the minimal angle between $\Pi \mathcal{C}$ and $\Pi(\mathcal{P} \cap \mathcal{Q})$ when $\mathcal{C}$ is a subspace satisfying (3.1-3.3). The next lemma gives conditions for a subspace $\mathcal{C}$ satisfying (3.1-3.3) to exist.

Lemma 3.2 Let $\mathcal{P}, \mathcal{K}, \mathcal{Q}, \mathcal{N}$ be given subspaces of a vector space $\mathcal{W}$. There exists a subspace $\mathcal{C}$ satisfying (3.1-3.3) if and only if

$$
\begin{aligned}
& \mathcal{N} \cap \mathcal{P} \subset \mathcal{K} \\
& \mathcal{N} \cap \mathcal{P} \cap \mathcal{Q}=\{0\} \\
& \mathcal{P}=\mathcal{K} \cap \mathcal{P}+\mathcal{Q} \cap \mathcal{P} .
\end{aligned}
$$

Proof 'Only if' (3.4) holds because otherwise (3.2) and (3.3) can not be satisfied together; (3.5) holds because of (3.3) and (3.1). To prove (3.6) note that $\mathcal{P}=\mathcal{P} \cap(\mathcal{C} \oplus \mathcal{P} \cap \mathcal{Q})=\mathcal{P} \cap \mathcal{C}+\mathcal{P} \cap \mathcal{Q} \subset \mathcal{K} \cap \mathcal{P}+\mathcal{Q} \cap \mathcal{P}$ and the reverse inclusion also follows since $\mathcal{K} \cap \mathcal{P} \subset \mathcal{P}$ and $\mathcal{Q} \cap \mathcal{P} \subset \mathcal{P}$.

'If' Let $\mathcal{V}$ be a subspace satisfying

$$
\mathcal{P} \cap \mathcal{Q} \cap \mathcal{K} \oplus \mathcal{N} \cap \mathcal{P} \oplus \mathcal{V}=\mathcal{K} \cap \mathcal{P}
$$

and $\mathcal{T}$ be a subspace such that

$$
\mathcal{T} \oplus(\mathcal{N}+\mathcal{P})=\mathcal{W} .
$$

Take $\mathcal{C}=\mathcal{N}+\mathcal{V}+\mathcal{T}$. It will be shown that $\mathcal{C}$ satisfies (3.1-3.3). We have $\mathcal{N} \subset \mathcal{C}$ and $\mathcal{P} \cap(\mathcal{N}+\mathcal{T})=$ $\mathcal{P} \cap(\mathcal{P}+\mathcal{N}) \cap(\mathcal{N}+\mathcal{T})=\mathcal{N} \cap \mathcal{P}$. Hence

$$
\begin{aligned}
\mathcal{P} \cap \mathcal{C} & =\mathcal{P} \cap(\mathcal{N}+\mathcal{V}+\mathcal{T})=\mathcal{V}+\mathcal{P} \cap(\mathcal{N}+\mathcal{T}) \\
& =\mathcal{V}+\mathcal{N} \cap \mathcal{P} \subset \mathcal{K} \cap \mathcal{P}
\end{aligned}
$$

which proves (3.2). On the other hand from (3.6) and (3.7) it follows that $\mathcal{P} \subset \mathcal{P} \cap \mathcal{Q}+\mathcal{N}+\mathcal{V}$. Hence $\mathcal{W}=\mathcal{P}+\mathcal{N}+\mathcal{T}=\mathcal{P} \cap \mathcal{Q}+\mathcal{N}+\mathcal{V}+\mathcal{T}=\mathcal{P} \cap \mathcal{Q}+\mathcal{C}$. Finally, from (3.8) and (3.7) it follows that $\mathcal{P} \cap \mathcal{Q} \cap \mathcal{C}=\mathcal{Q} \cap \mathcal{K} \cap \mathcal{P} \cap(\mathcal{V}+\mathcal{N} \cap \mathcal{P})=\{0\}$ which proves $(3.1)$. 
Remark 3.3 It can be shown that for the subspaces $\mathcal{P}, \mathcal{K}, \mathcal{Q}, \mathcal{N}$ defined in the previous section (3.4) and (3.5) are equivalent to

$$
\operatorname{ker}\left[\begin{array}{cc}
s I-A & -E \\
C & 0
\end{array}\right] \subset \operatorname{ker}\left[\begin{array}{cc}
D & 0
\end{array}\right], \quad \operatorname{ker}\left[\begin{array}{c}
s I-A \\
C
\end{array}\right] \subset \operatorname{ker} D
$$

respectively. It should be noted that (3.5) follows from the detectability of the pair $(C, A)$ and is redundant. Under the stabilizability assumption of the pair $(A, B)(3.6)$ is equivalent to

$$
\operatorname{im}\left[\begin{array}{c}
E \\
0
\end{array}\right] \subset \operatorname{im}\left[\begin{array}{cc}
s I-A & -B \\
D & 0
\end{array}\right] .
$$

It is well known [13] that DDIS is solvable if and only if the pair $(A, B)$ is stabilizable, $(C, A)$ is detectable and the linear matrix equation

$$
\left[\begin{array}{cc}
s I-A & -E \\
D & 0
\end{array}\right]=\left[\begin{array}{cc}
s I-A & -B \\
D & 0
\end{array}\right] X(s)\left[\begin{array}{cc}
s I-A & -E \\
C & 0
\end{array}\right]
$$

admits a solution over $R H_{\infty}$. Clearly (3.4-3.6) are necessary conditions for the solvability of DDIS which are however not sufficient.

Note that for a subspace $\mathcal{C}$ satisfying $\mathcal{N} \subset \mathcal{C}(3.1)$ and (3.2) can be written in terms of $\Pi \mathcal{C}$ as

$$
\begin{aligned}
& \Pi \mathcal{C} \oplus \Pi(\mathcal{P} \cap \mathcal{Q})=\mathcal{Y} \times \mathcal{U} \\
& \Pi \mathcal{C} \cap \Pi \mathcal{P} \subset \Pi(\mathcal{K} \cap \mathcal{P}) .
\end{aligned}
$$

It also follows from (3.10) that

$$
\Pi \mathcal{C} \cap \Pi \mathcal{P} \oplus \Pi(\mathcal{P} \cap \mathcal{Q})=\Pi \mathcal{P} .
$$

Conditions $(3.11,3.12)$ on the subspace $\Pi \mathcal{C} \cap \Pi \mathcal{P}$ suggest the problem of finding the maximal value of the minimal angle between two complementary subspaces when one of the subspaces is contained in another subspace. This is considered in the following lemma.

Lemma 3.4 Let $\mathcal{R}, \mathcal{S}, \mathcal{T}$ be subspaces of a unitary space satisfying $\mathcal{R}+\mathcal{S}=\mathcal{T}$. Then

$$
\max _{\mathcal{V}}\{\phi(\mathcal{V}, \mathcal{R}) \mid \mathcal{V} \subset \mathcal{S}, \mathcal{V} \oplus \mathcal{R}=\mathcal{T}\}=\phi\left((\mathcal{R} \cap \mathcal{S})^{\perp} \cap \mathcal{S}, \mathcal{R}\right) .
$$

PROOF Since angles between subspaces are unchanged by unitary transformations and image representations of subspaces are determined up to right multiplication by nonsingular matrices we may as well assume that

$$
\mathcal{R} \cap \mathcal{S}=\operatorname{im}\left[\begin{array}{l}
I \\
0
\end{array}\right], \mathcal{R}=\operatorname{im}\left[\begin{array}{cc}
I & 0 \\
0 & R_{1}
\end{array}\right] \quad \text { and } \mathcal{S}=\operatorname{im}\left[\begin{array}{cc}
I & 0 \\
0 & S_{1}
\end{array}\right]
$$

where $R_{1}$ and $S_{1}$ are full column rank matrices of appropriate sizes. All subspaces $\mathcal{V}$ that satisfy $\mathcal{V} \subset \mathcal{S}, \quad \mathcal{V} \oplus \mathcal{R}=\mathcal{T}$ are then of the form $\mathcal{V}=\operatorname{im} \operatorname{col}\left(V_{1}, S_{1}\right)$ for some matrix $V_{1}$. Let $\left[\begin{array}{ll}0 & \tilde{R}_{1}\end{array}\right]$ be a normalized kernel representation for $\mathcal{R}$ then by [17, Prop. 2.4]

$$
\begin{aligned}
\sin \phi(\mathcal{V}, \mathcal{R}) & =\sigma_{\min }\left\{\left[\begin{array}{ll}
0 & \tilde{R}_{1}
\end{array}\right]\left[\begin{array}{c}
V_{1} \\
S_{1}
\end{array}\right]\left(V_{1}^{*} V_{1}+S_{1}^{*} S_{1}\right)^{-1 / 2}\right\} \\
& =\min _{x \neq 0} \frac{\left\|\tilde{R}_{1} S_{1}\left(V_{1}^{*} V_{1}+S_{1}^{*} S_{1}\right)^{-1 / 2} x\right\|}{\|x\|} .
\end{aligned}
$$


Making the change of variables $y=\left(V_{1}^{*} V_{1}+S_{1}^{*} S_{1}\right)^{-1 / 2} x$ and writing $\|x\|=\left(\left\|V_{1} y\right\|^{2}+\left\|S_{1} y\right\|^{2}\right)^{1 / 2}$ we have

$$
\begin{aligned}
\sin \phi(\mathcal{V}, \mathcal{R}) & =\min _{y \neq 0} \frac{\left\|\tilde{R}_{1} S_{1} y\right\|}{\left(\left\|V_{1} y\right\|^{2}+\left\|S_{1} y\right\|^{2}\right)^{1 / 2}} \\
& \leq \min _{y \neq 0} \frac{\left\|\tilde{R}_{1} S_{1} y\right\|}{\left\|S_{1} y\right\|}=\sigma_{\min }\left\{\tilde{R}_{1} S_{1}\left(S_{1}^{*} S_{1}\right)^{-1 / 2}\right\}
\end{aligned}
$$

But the upper bound is attained by $\mathcal{V}=\operatorname{im}\left[\begin{array}{c}0 \\ S_{1}\end{array}\right]=(\mathcal{R} \cap \mathcal{S})^{\perp} \cap \mathcal{S}$ which establishes the conclusion.

Applying Lemma 3.4 with $\Pi(\mathcal{P} \cap \mathcal{Q}), \Pi(\mathcal{K} \cap \mathcal{P})$ and $\Pi \mathcal{P}$ playing the roles of $\mathcal{R}, \mathcal{S}$ and $\mathcal{T}$ respectively leads to the main result for the minimal angle between $\Pi \mathcal{C}$ and $\Pi(\mathcal{P} \cap \mathcal{Q})$.

TheOREM 3.5 Define subspaces $\mathcal{Q}, \mathcal{K}, \mathcal{N}$ of the vector space $\mathcal{W}$ by (2.16-2.18) and let $\mathcal{P}$ be a subspace satisfying (3.4-3.6). Let $\Pi: \mathcal{W} \mapsto \mathcal{Y} \times \mathcal{U}$ be the projection onto $\mathcal{K} \cap \mathcal{Q}$ along $\mathcal{N}$ and $\mathcal{V}_{0}=(\Pi(\mathcal{P} \cap \mathcal{K} \cap$ $\mathcal{Q}))^{\perp} \cap \Pi(\mathcal{P} \cap \mathcal{K})$. Under these conditions for any subspace $\mathcal{C}$ satisfying (3.1-3.3)

$$
\sin \phi\left(\Pi \mathcal{C}, \Pi(\mathcal{P} \cap \mathcal{Q}) \leq \sin \phi\left(\mathcal{V}_{0}, \Pi(\mathcal{P} \cap \mathcal{Q})\right)\right.
$$

and equality is achieved for instance by

$$
\Pi \mathcal{C}=\mathcal{V}_{0}+(\Pi \mathcal{P})^{\perp}
$$

Proof It follows from the definition of the minimal angle that $\phi(\Pi \mathcal{C}, \Pi(\mathcal{P} \cap \mathcal{Q})) \leq \phi(\Pi \mathcal{C} \cap \Pi \mathcal{P}, \Pi(\mathcal{P} \cap$ $\mathcal{Q})$ ). So $(3.15)$ follows from $(3.11,3.12)$ and Lemma 3.4.

Next, we prove that the upper bound is indeed attained by the choice (3.16). This follows by noting that $(\Pi \mathcal{P})^{\perp}$ is orthogonal to $\Pi(\mathcal{P} \cap \mathcal{Q})$ and hence $\phi(\Pi \mathcal{C}, \Pi(\mathcal{P} \cap \mathcal{Q}))=\phi\left(\mathcal{V}_{0}, \Pi(\mathcal{P} \cap \mathcal{Q})\right)$.

Finally, note that the subspace $\mathcal{C}$ satisfying $\mathcal{N} \subset \mathcal{C}$ and $(3.16)$ is uniquely determined as $\mathcal{C}=\Pi^{-1}(\Pi \mathcal{C})$. It remains to verify that $\mathcal{C}$ satisfies $(3.1,3.2)$ or equivalently $\Pi \mathcal{C}$ satisfies $(3.10,3.11)$. We have

$$
\Pi \mathcal{C} \cap \Pi \mathcal{P}=\left(\mathcal{V}_{0}+(\Pi \mathcal{P})^{\perp}\right) \cap \Pi \mathcal{P}=\mathcal{V}_{0} \subset \Pi(\mathcal{K} \cap \mathcal{P})
$$

which proves (3.11). On the other hand, $\Pi \mathcal{C} \cap \Pi(\mathcal{P} \cap \mathcal{Q})=\mathcal{V}_{0} \cap \Pi(\mathcal{P} \cap \mathcal{Q})=\{0\}$ and from (3.6) it follows that $\mathcal{V}_{0}+\Pi(\mathcal{P} \cap \mathcal{Q})=\Pi(\mathcal{K} \cap \mathcal{P})+\Pi(\mathcal{P} \cap \mathcal{Q})=\Pi \mathcal{P}$. Hence $\Pi \mathcal{C}+\Pi(\mathcal{P} \cap \mathcal{Q})=\Pi \mathcal{W}$ establishing $(3.10)$.

The relation (3.15) gives an upper bound on the minimal angle for each $s \in \mathbb{C}^{+}$. An overall bound for the minimum of the left hand side can be obtained by finding the greatest lower bound of the right hand side. In general, the subspace $\mathcal{V}_{0}$ is not a continuous function of $s$. So the minimum of the right hand side of (3.15) on $\mathbb{C}^{+}$may not exist and it may not be sufficient to search along the imaginary axis to find the infimum.

In [8] it is shown that the maximum of the robustness measure (2.27) over all stabilizing controllers is given by

$$
\sqrt{1-\left\|\Gamma_{\tilde{M}} *\right\|^{2}}
$$

where $\tilde{M}(s)$ is a normalized kernel representation of the subspace valued function $\Pi(\mathcal{P}(s) \cap \mathcal{Q})$ and $\Gamma_{\tilde{M}^{*}}$ is the Hankel operator with symbol $\tilde{M}^{T}(-s)[5]$. Hence by Thm. 3.5 one may conclude that a necessary condition for the solvability of $\operatorname{DDRS}(\gamma)$ is

$$
\gamma \leq \min \left\{\inf _{s \in \mathbb{C}^{+}} \sin \phi\left(\mathcal{V}_{0}(s), \Pi(\mathcal{P}(s) \cap \mathcal{Q})\right), \sqrt{1-\left\|\Gamma_{\tilde{M}^{*}}\right\|^{2}}\right\}
$$


In section 5 it will be shown by an example that the actual robustness of stability that can be achieved by a solution of DDIS is in general smaller than the upper bound given in (3.18). However this result should be anticipated since the assumptions (3.4-3.6) from which the upper bound (3.15) is derived do not even imply the solvability of DDIS and if DDIS is not solvable (3.15) does not have any meaning at all.

4. Solution of the disturbance DeCoupling PRoblem With Robust Stability

In this section we solve DDRS by parametrizing all subspace valued functions satisfying the conditions of Theorem 3.1 and expressing the projection operator on $\Pi \mathcal{C}(s)$ along $\Pi(\mathcal{P}(s) \cap \mathcal{Q})$ in terms of a free parameter. The maximization of the robustness measure $(2.27)$ then reduces to a standard $H_{\infty}$ optimization problem. To simplify the exposition, we shall work under the following assumption.

Assumption 4.1 The transfer matrix $G_{12}(s)=D(s I-A)^{-1} B$ from control inputs to controlled outputs is left invertible as a matrix over the rational functions.

Since the disturbance decoupling problem is generically not solvable if the number of controlled outputs exceeds the number of control inputs [21, p. 92], Assumption 4.1 effectively restricts the discussion to systems in which the number of controlled outputs equals the number of control inputs. If there are more control inputs than controlled outputs, the problem becomes easier in a sense but messier from a mathematical point of view since we get extra degrees of freedom in the parametrization. The first lemma gives an equivalent condition for the left invertibility of $G_{12}(s)$ in terms of the subspace valued functions introduced above.

LEMMA 4.2 The transfer function $G_{12}(s)$ is left invertible as a matrix over the rational functions if and only if $\mathcal{K} \cap \mathcal{P}(s) \cap \mathcal{Q}=\{0\}$ for some $s \in \mathbb{C}^{+}$.

Proof From $(2.16,2.17)$ and (2.13) it follows that $w=\left[\begin{array}{lll}0 & y & 0 u\end{array}\right]^{T} \in \mathcal{K} \cap \mathcal{P}(s) \cap \mathcal{Q}$ if and only if there exists $x$ such that

$$
\left[\begin{array}{ccc}
s I-A & 0 & -B \\
D & 0 & 0 \\
C & -I & 0
\end{array}\right]\left[\begin{array}{l}
x \\
y \\
u
\end{array}\right]=0 .
$$

Hence, since the pair $(C, A)$ is detectable, $\mathcal{K} \cap \mathcal{P}(s) \cap \mathcal{Q}=\{0\}$ for $s \in \mathbb{C}^{+}$if and only if $\left[\begin{array}{cc}s I-A & -B \\ D & 0\end{array}\right]$ has full column rank. By a standard result, see [23, Lemma 3.33] for instance, this is equivalent to the left invertibility of $G_{12}(s)$.

Throughout this section we shall be working with image and kernel representations of the subspace valued functions introduced in section 2. For the stabilizable and detectable system (2.1-2.3) with subspace valued function $\mathcal{P}(s)$ it follows from [4] that there exist $P(s) \in R H_{\infty}^{q \times(m+r)}$ and $\tilde{P}(s) \in$ $R H_{\infty}^{(p+\ell) \times q}$ which have respectively full column rank and full row rank everywhere on $\mathbb{C}^{+}$such that

$$
\mathcal{P}(s)=\operatorname{im} P(s)=\operatorname{ker} \tilde{P}(s) \text { for all } s \in \mathbb{C}^{+} .
$$

Let $N(s) D^{-1}(s)=\tilde{D}(s)^{-1} \tilde{N}(s)$ be respectively a right coprime and a left coprime factorization over $R H_{\infty}$ of the transfer matrix of the system (2.1-2.3). Appropriate matrices $P(s)$ and $\tilde{P}(s)$ satisfying (4.1) are given by $P(s)=\operatorname{col}(N(s), D(s))$ and $\tilde{P}(s)=[\tilde{D}(s)-\tilde{N}(s)]$. Similarly one obtains image and kernel representations of the subspace valued function of the controller from right and left coprime factorizations of its transfer matrix. For the subspaces $\mathcal{K}$ and $\mathcal{N}$ defined by $(2.17-2.18)$ we take full rank matrices $\tilde{K}$ and $N$ such that

$$
\mathcal{K}=\operatorname{ker} \tilde{K}, \quad \mathcal{N}=\operatorname{im} N .
$$

It follows from the next lemma that representations of subspace valued functions are not unique but all image (kernel) representations are right (left) associates of each other. A related result on coprime fractions of a rational function is given in [18, Thm. 4.1.43]. 
LEMMA 4.3 Assume that $T_{1}(s)$ and $T_{2}(s)$ are $R H_{\infty}$ matrices such that $T_{1}(s)$ has full row rank everywhere on $\mathbb{C}^{+}$and $\operatorname{ker} T_{1}(s) \subset \operatorname{ker} T_{2}(s)$ for all $s \in \mathbb{C}^{+}$. Then there exists an $R H_{\infty}$ matrix $H(s)$ satisfying $H(s) T_{1}(s)=T_{2}(s)$.

Proof Let $T_{0}(s)$ be an $R H_{\infty}$ matrix such that $U(s)=\operatorname{col}\left(T_{0}(s), T_{1}(s)\right)$ is unimodular. Write $U^{-1}(s)=\left[R_{0}(s) R_{1}(s)\right]$ so that $\operatorname{ker} T_{1}(s)=\operatorname{im} R_{0}(s)$ for all $s$ in $\mathbb{C}^{+}$. Then we have $T_{2}(s) R_{0}(s)=0$ hence

$$
T_{2}(s)=T_{2}(s)\left[\begin{array}{ll}
R_{0}(s) & R_{1}(s)
\end{array}\right]\left[\begin{array}{l}
T_{0}(s) \\
T_{1}(s)
\end{array}\right]=H(s) T_{1}(s)
$$

with $H(s)=T_{2}(s) R_{1}(s)$.

In the sequel we shall also need representations of the subspace valued function $\mathcal{P}(s) \cap \mathcal{Q}$. These can be obtained using the following lemma.

LEMMA 4.4 Let the subspace valued function $\mathcal{P}(s)$ be defined by (2.13) and the subspace $\mathcal{Q}$ be defined by (2.16). There exists an $R H_{\infty}$ matrix $P_{u}(s)$ of size $q \times m$ and an $R H_{\infty}$ matrix $\tilde{P}_{u}(s)$ of size $(p+r+\ell) \times q$ such that

$$
\mathcal{P}(s) \cap \mathcal{Q}=\operatorname{im} P_{u}(s)=\operatorname{ker} \tilde{P}_{u}(s) \quad \text { for all } s \in \mathbb{C}^{+} .
$$

Moreover, if $\tilde{P}(s)$ is a kernel representation for the subspace valued function $\mathcal{P}(s)$ there exists a $(p+\ell) \times(p+\ell+r)$ matrix $\tilde{H}(s)$ in $R H_{\infty}$ such that $\tilde{H}(s)$ has full row rank for all $s$ in $\mathbb{C}^{+}$and $\tilde{P}(s)=\tilde{H}(s) \tilde{P}_{u}(s)$.

Proof Let $\tilde{Q}$ and $\tilde{P}(s)$ be full row rank matrices such that $\mathcal{Q}=\operatorname{ker} \tilde{Q}$ and $\mathcal{P}(s)=\operatorname{ker} \tilde{P}(s)$ for all $s \in \mathbb{C}^{+}$. Then $\tilde{P}_{u}(s)=\operatorname{col}(\tilde{P}(s), \tilde{Q})$ is a kernel representation for $\mathcal{P}(s) \cap \mathcal{Q}$. Since $\operatorname{dim}(\mathcal{P}(s) \cap \mathcal{Q})=m$ for all $s \in \mathbb{C}^{+}, \tilde{P}_{u}(s)$ has full row rank everywhere on $\mathbb{C}^{+}$. Hence there exists a unimodular matrix $U(s)$ such that $\tilde{P}_{u}(s) U(s)=\left[\begin{array}{ll}I & 0\end{array}\right]$. Let $P_{u}(s)$ denote the last $m$ columns of $U(s)$ then $\operatorname{im} P_{u}(s) \subset \operatorname{ker} \tilde{P}_{u}(s)$ and it follows by counting dimensions that we have indeed $\mathcal{P}(s) \cap \mathcal{Q}=\operatorname{im} P_{u}(s)$ for all $s \in \mathbb{C}^{+}$. The claim about the existence of $\tilde{H}(s)$ satisfying $\tilde{P}(s)=\tilde{H}(s) \tilde{P}_{u}(s)$ follows from Lemma 4.3. $\tilde{H}(s)$ must have full row rank for all $s$ in $\mathbb{C}^{+}$because otherwise $\tilde{P}(s)$ would not have this property.

Now, assume that disturbance decoupling problem with internal stability is solvable and let $C_{0}(s)$ be an image representation of a particular solution. Take image and kernel representations of the subspace valued functions $\mathcal{P}(s)$ and $\mathcal{P}(s) \cap \mathcal{Q}$ as in $(4.1,4.3)$. Since $\operatorname{im} C_{0}(s)$ and $\operatorname{ker} \tilde{P}_{u}(s)$ are complementary for all $s \in \mathbb{C}^{+}$the matrix function $\tilde{P}_{u}(s) C_{0}(s)$ is a unit in $R H_{\infty}$. As image representations are unique up to multiplication by unimodular matrices from the right we may assume that $C_{0}(s)$ is normalized such that

$$
\tilde{P}_{u}(s) C_{0}(s)=I \text {. }
$$

From the Kučera-Youla parametrization [11, 22] it follows that image representation of all stabilizing controllers can be written in the form

$$
C(s)=C_{0}(s)-P_{u}(s) \Psi(s)
$$

where $\Psi(s)$ is an arbitrary $m \times(p+r+\ell)$ matrix in $R H_{\infty}$. At this point we need the following lemma from [3] to derive the additional condition on the free parameter $\Psi(s)$ for im $C(s)$ to satisfy (3.2) corresponding to the disturbance decoupling requirement.

LEMmA 4.5 Let $\mathcal{W}$ be a vector space and let $\mathcal{C}, \mathcal{P}$ and $\mathcal{M}$ be subspaces of $\mathcal{W}$ such that $\mathcal{C} \oplus \mathcal{P}=\mathcal{W}$ and $\mathcal{P} \subset \mathcal{M}$. Denoting the projection on $\mathcal{C}$ along $\mathcal{P}$ by $\Pi_{\mathcal{C}}^{\mathcal{P}}$ we have $\mathcal{C} \cap \mathcal{M}=\Pi_{\mathcal{C}}^{\mathcal{P}} \mathcal{M}$. 
Because of the normalization condition (4.4) the projection on $\mathcal{C}(s)$ along $\mathcal{P}(s) \cap \mathcal{Q}$ is given by $C(s) \tilde{P}_{u}(s)$ and $(3.2)$ can be written in the form

$$
C(s) \tilde{P}_{u}(s) \operatorname{ker} \tilde{P}(s) \subset \mathcal{K} \text { for all } s \in \mathbb{C}^{+} .
$$

Since $\tilde{P}_{u}(s)$ has full row rank for all $s \in \mathbb{C}^{+}$and there exists a matrix $\tilde{H}(s)$ such that $\tilde{P}(s)=\tilde{H}(s) \tilde{P}_{u}(s)$, (4.6) is equivalent to

$$
C(s) \operatorname{ker} \tilde{H}(s) \subset \mathcal{K} \text { for all } s \in \mathbb{C}^{+} .
$$

Substituting (4.5) into (4.7) and noting that $C_{0}(s)$ ker $\tilde{H}(s) \subset \mathcal{K}$ for all $s \in \mathbb{C}^{+}$as $C_{0}(s)$ is an image representation of a particular solution of (3.1-3.3) we get

$$
P_{u}(s) \Psi(s) \operatorname{ker} \tilde{H}(s) \subset \mathcal{K} \text { for all } s \in \mathbb{C}^{+} .
$$

Under the assumption 4.1 the matrix $\tilde{K} P_{u}(s)$ is left invertible and (4.8) simplifies to

$$
\Psi(s) \operatorname{ker} \tilde{H}(s)=\{0\} \quad \text { for all } s \in \mathbb{C}^{+} .
$$

By Lemma 4.3 the general solution of (4.9) for $\Psi(s)$ is given by

$$
\Psi(s)=\Theta(s) \tilde{H}(s)
$$

where $\Theta(s) \in R H_{\infty}^{m \times(p+\ell)}$ is a free parameter.

Using the projection operator $C(s) \tilde{P}_{u}(s)$ the realizability condition (3.3) can be written as

$$
C(s) \tilde{P}_{u}(s) \mathcal{N}=\mathcal{N} \text { for all } s \in \mathbb{C}^{+} .
$$

Recalling again that $C_{0}(s)$ is an image representation of a particular solution normalized as in (4.4) and $P_{u}(s)$ has full column rank, (4.11) leads to the following condition on the free parameter $\Psi(s)$ :

$$
\Psi(s) \tilde{P}_{u}(s) \mathcal{N}=\{0\} \text { for all } s \in \mathbb{C}^{+} .
$$

The general solution of all $R H_{\infty}$ functions $\Psi(s)$ that satisfy (4.10) and (4.12) is of the form

$$
\Psi(s)=\Omega(s) T(s) \tilde{H}(s)
$$

where $T(s)$ is a matrix whose rows constitute a basis for the $R H_{\infty}$ module spanned by the vectors $t(s)$ satisfying $t(s) \tilde{P}(s) N=0$. Note that $T(s)$ has full row rank for all $s \in \mathbb{C}^{+}$and can be found using for instance the unimodular matrices that transform $\tilde{P}(s) N$ to Hermite or Smith form. Substituting (4.13) into (4.5) we finally obtain a parametrization of all solutions of the disturbance decoupling problem with stability under the assumption 4.1. This is summarized in the following theorem.

THEOREM 4.6 Consider the system (2.1-2.3) with the assumption 4.1. Let $P_{u}(s)$ and $\tilde{P}_{u}(s)$ be respectively image and kernel representations for $\mathcal{P}(s) \cap \mathcal{Q}$ and $\tilde{P}(s)$ be a kernel representation for the subspace valued function $\mathcal{P}(s)$. Define $\tilde{H}(s)$ such that $\tilde{P}(s)=\tilde{H}(s) \tilde{P}_{u}(s)$. Let $T(s)$ be a matrix whose rows form a basis for the $R H_{\infty}$ module spanned by the vectors $t(s)$ satisfying $t(s) \tilde{P}(s) N=0$. Assume that DDIS is solvable and let $C_{0}(s)$ be an image representation of the subspace valued functions of a particular solution normalized such that $\tilde{P}_{u}(s) C_{0}(s)=I$. Under these conditions the general form of an image representation $C(s)$ of a solution of DDIS is given by

$$
C(s)=C_{0}(s)-P_{u}(s) \Omega(s) T(s) \tilde{H}(s)
$$

where $\Omega(s)$ is a free parameter in $R H_{\infty}$.

In order to determine the optimal robustness of stability that can be achieved by a solution of the disturbance decoupling problem, we compute the projection operator $\Gamma(s)$ on $\Pi \mathcal{C}(s)$ along $\Pi(\mathcal{P}(s) \cap$ $\mathcal{Q})$ and use the characterization $(2.26)$ of the minimal angle to convert the problem to a standard $H_{\infty}$ optimization. The next lemma establishes the relationship between the projection operators corresponding to the direct sum decompositions (2.21) and (2.24). 
LEMmA 4.7 Let $\mathcal{P}, \mathcal{Q}$ and $\mathcal{C}$ be subspaces of $\mathcal{W}$ satisfying (3.1, 3.3). Let $\Lambda$ denote the projection on $\mathcal{C}$ along $\mathcal{P} \cap \mathcal{Q}$ and $\Gamma$ be the projection on $\Pi \mathcal{C}$ along $\Pi(\mathcal{P} \cap \mathcal{Q})$. Then we have $\Gamma=\Pi \Lambda \Pi^{\dagger}$ where $\Pi^{\dagger}$ is a right inverse of $\Pi$.

Proof Take $w \in \mathcal{W}$. There exist unique vectors $c \in \mathcal{C}$ and $p \in \mathcal{P} \cap \mathcal{Q}$ such that $w=c+p$. Hence $\Gamma(\Pi w)=\Pi(\Lambda w)=\Pi c$ which proves that $\Gamma \Pi=\Pi \Lambda$. The conclusion follows by applying $\Pi^{\dagger}$ on both sides.

Using this lemma the projection $\Gamma(s)$ is readily calculated as

$$
\begin{aligned}
\Gamma(s) & =\Pi C(s) \tilde{P}_{u}(s) \Pi^{\dagger} \\
& =\Pi C_{0}(s) \tilde{P}_{u}(s) \Pi^{\dagger}-\Pi P_{u}(s) \Omega(s) T(s) \tilde{P}(s) \Pi^{\dagger}
\end{aligned}
$$

where we have used (4.14) for $C(s)$ and the fact $\tilde{P}(s)=\tilde{H}(s) \tilde{P}_{u}(s)$. Thus the maximum value of $\gamma$ for which the problem $\operatorname{DDRS}(\gamma)$ is solvable is given by

$$
\gamma_{\max }=\left\{\min _{\Omega(s) \in R H_{\infty}}\left\|\Pi C_{0}(s) \tilde{P}_{u}(s) \Pi^{\dagger}-\Pi P_{u}(s) \Omega(s) T(s) \tilde{P}(s) \Pi^{\dagger}\right\|_{\infty}\right\}^{-1} .
$$

REMARK 4.8 To see that we have indeed a maximum for the stability margin $\gamma$, note that for all $s \in \mathbb{C}^{+}$ we have $\mathcal{N} \cap \mathcal{P}(s) \cap \mathcal{Q}=\{0\}$ hence $\operatorname{rank} \Pi P_{u}(s)=\operatorname{rank} P_{u}(s)-\operatorname{dim}(\mathcal{N} \cap \mathcal{P}(s) \cap \mathcal{Q})=m$. On the other hand since $T(s) \tilde{P}(s)$ has full row rank, $\left[\begin{array}{ll}\Pi^{\dagger} & N\end{array}\right]$ is nonsingular and $T(s) \tilde{P}(s)\left[\begin{array}{ll}\Pi^{\dagger} & N\end{array}\right]=\left[\begin{array}{ll}T(s) \tilde{P}(s) \Pi^{\dagger} & 0\end{array}\right]$, $T(s) \tilde{P}(s) \Pi^{\dagger}$ has full row rank for all $s \in \mathbb{C}^{+}$as well. Therefore by [5, Thm. 6.1] the maximum is attained in (4.17).

This leads to the main result for the solution of $\operatorname{DDRS}(\gamma)$ under the assumption 4.1.

THEOREM 4.9 Consider the system under the assumption 4.1 and assume that disturbance decoupling problem with internal stability is solvable. Define $R H_{\infty}$ matrix functions $\tilde{P}(s), \tilde{P}_{u}(s), P_{u}(s), C_{0}(s)$ and $T(s)$ as in Theorem 4.6 and $\gamma_{\max }$ by (4.17). The problem DDRS( $\left.\gamma\right)$ is solvable if and only if

$$
\gamma \leq \gamma_{\max }
$$

ProOF It has already been shown that $\gamma_{\max }$ is the maximum stability margin that can be achieved by a solution of the disturbance decoupling problem with stability. So (4.18) is clearly necessary. When (4.18) is satisfied an image representation of the subspace valued function of a solution to $\operatorname{DDRS}(\gamma)$ is given by (4.14) where the free parameter $\Omega(s)$ is chosen as the solution of the $H_{\infty}$ minimization problem $\left\|\Pi C_{0}(s) \tilde{P}_{u}(s) \Pi^{\dagger}-\Pi P_{u}(s) \Omega(s) T(s) \tilde{P}(s) \Pi^{\dagger}\right\|_{\infty} \leq \gamma^{-1}$.

In the next section the construction of a solution to $\operatorname{DDRS}(\gamma)$ is illustrated by an example.

\section{EXAmPle}

Consider the system described by

$$
\begin{aligned}
\dot{x}(t) & =\left[\begin{array}{ll}
0 & -1 \\
1 & -\alpha
\end{array}\right] x(t)+\left[\begin{array}{l}
1 \\
1
\end{array}\right] u(t)+\left[\begin{array}{l}
1 \\
0
\end{array}\right] d(t) \\
z(t) & =\left[\begin{array}{ll}
0 & 1
\end{array}\right] x(t) \\
y(t) & =x(t)
\end{aligned}
$$

The system represents a second order plant with damping ratio $\alpha / 2$ subject to the unknown disturbance $d(t)$. It is desired to decouple the disturbance from the controlled output $z(t)$ and at the same time stabilize the system by applying a feedback to the measured output $y(t)$ which is assumed to be the whole state vector. We first compute the upper bound on robustness of stability derived by the pointwise geometrical analysis in Section 3 and then compare this with the overall bound of Glover 
and McFarlane [8] and with the actual robustness of stability that can be achieved by a solution of the disturbance decoupling problem.

The transfer matrix of the system is given by

$$
\left[\begin{array}{ll}
G_{11}(s) & G_{12}(s) \\
G_{21}(s) & G_{22}(s)
\end{array}\right]=\frac{1}{s^{2}+\alpha s+1}\left[\begin{array}{cc}
1 & s+1 \\
s+\alpha & s+\alpha-1 \\
1 & s+1
\end{array}\right]
$$

Note that $G_{12}(s)$ satisfies the left invertibility assumption. A kernel representation of the subspace valued function $\mathcal{P}(s)$ can be obtained by eliminating the $x$ variables from the equations (2.11) which, after dividing by $s+1$, gives

$$
\tilde{P}(s)=\frac{1}{s+1}\left[\begin{array}{ccccc}
0 & s & 1 & -1 & -1 \\
0 & -1 & s+\alpha & 0 & -1 \\
s+1 & 0 & -s-1 & 0 & 0
\end{array}\right]
$$

Image and kernel representations of the subspace valued function $\mathcal{P}(s) \cap \mathcal{Q}$ are of the form

$$
P_{u}(s)=\frac{1}{(s+1)^{2}}\left[\begin{array}{lllll}
s+1 & s+\alpha-1 & s+1 & 0 & s^{2}+\alpha s+1
\end{array}\right]^{T}, \quad \tilde{P}_{u}(s)=\left[\begin{array}{c}
\tilde{P}(s) \\
\tilde{Q}
\end{array}\right]
$$

where $\tilde{Q}$ is the fourth row of the $5 \times 5$ unit matrix. Note that $\tilde{P}(s)=\tilde{H} \tilde{P}_{u}(s)$ where $\tilde{H}=\left[\begin{array}{ll}I_{3} & 0\end{array}\right]$. The subspace valued function $\mathcal{V}_{0}(s)$ used in the computation of the upper bound for the minimal angle is then given by

$$
\mathcal{V}_{0}(s)=\Pi(\mathcal{P}(s) \cap \mathcal{K})=\operatorname{im}\left[\begin{array}{lll}
1 & 0 & -1
\end{array}\right]^{T} \quad \operatorname{Re}(s) \geq 0
$$

while for $s=\infty$ we have

$$
\mathcal{V}_{0}(\infty)=\{0\}
$$

Therefore (3.15) gives the trivial bound 1 for $s=\infty$, and for $\operatorname{Re}(s) \geq 0$ the minimal angle between $\Pi \mathcal{C}(s)$ and $\Pi(\mathcal{P}(s) \cap \mathcal{Q})$ is bounded by

$$
\sin \phi\left(\mathcal{V}_{0}(s), \Pi(\mathcal{P}(s) \cap \mathcal{Q})\right)=\sin \phi\left(\operatorname{im}\left[\begin{array}{c}
1 \\
0 \\
-1
\end{array}\right], \operatorname{im}\left[\begin{array}{c}
s+\alpha-1 \\
s+1 \\
s^{2}+\alpha s+1
\end{array}\right]\right)
$$

In the present case the infimum of the expression (5.9) for $\operatorname{Re}(s) \geq 0$ can be found by a 1-dimensional search along the imaginary axis. In Figure 1 this is plotted as the dashed curve for $-2 \leq \alpha \leq 2$ and compared with the optimal robustness of stability without taking the disturbance decoupling requirement into account.

The rest of the example is devoted to find the actual robustness of stability $\gamma_{\max }$ that can be attained by a solution of the disturbance decoupling problem. A particular solution of the DDIS is the constant state feedback

$$
u(t)=\left[\begin{array}{ll}
-1 & \alpha-1
\end{array}\right] y(t)
$$

The image representation of this controller satisfying the normalization condition $\tilde{P}_{u}(s) C_{0}(s)=I$ is given by

$$
C_{0}(s)=\frac{1}{s+1}\left[\begin{array}{cccc}
0 & s+1 & s+1 & 0 \\
s+1 & \alpha-2 & 0 & 1 \\
0 & s+1 & 0 & 0 \\
0 & 0 & 0 & s+1 \\
-s-1 & \alpha s-s+1 & 0 & -1
\end{array}\right] .
$$


An $R H_{\infty}$ matrix $T(s)$ containing a maximal number of linearly independent rows and satisfying $T(s) \tilde{P}(s) N=0$ is taken as

$$
T(s)=\left[\begin{array}{lll}
0 & 1 & 0
\end{array}\right] .
$$

Now, the projection $\Gamma(s)$ on $\Pi \mathcal{C}(s)$ along $\Pi(\mathcal{P}(s) \cap \mathcal{Q})$ can be computed from (4.16) where

$$
\begin{aligned}
& {\left[\begin{array}{cc}
\Pi C_{0}(s) \tilde{P}_{u}(s) \Pi^{\dagger} & \Pi P_{u}(s) \\
T(s) \tilde{P}(s) \Pi^{\dagger} & 0
\end{array}\right]=} \\
& \frac{1}{(s+1)^{2}}\left[\begin{array}{ccc|c}
s^{2}+s+2-\alpha & (\alpha-1)(s+\alpha-1) & 1-s-\alpha & s+\alpha-1 \\
-s-1 & s^{2}+(\alpha+1) s+\alpha & -s-1 & s+1 \\
-s^{2}-\alpha s-1 & (\alpha-1)\left(s^{2}+\alpha s+1\right) & (2-\alpha) s & s^{2}+\alpha s+1 \\
\hline-s-1 & s^{2}+(\alpha+1) s+\alpha & -s-1 & 0
\end{array}\right]
\end{aligned}
$$

The minimum of $\|\Gamma(s)\|_{\infty}$ over all $\Omega(s) \in R H_{\infty}$ is computed by the loop shifting, two Riccati formula of $H_{\infty}$ optimization via gamma iteration [15], [23, Ch. 17] giving

$$
\gamma_{\max }=\min _{\Omega(s) \in R H_{\infty}}\|\Gamma(s)\|_{\infty}^{-1}
$$

as the maximum robustness of stability that can be achieved by a solution of the disturbance decoupling problem. In Figure 1 this is shown by the solid curve for $-2 \leq \alpha \leq 2$. Comparing the upper bound obtained by the pointwise geometrical analysis and the overall bound of Glover, McFarlane with the actual achievable robustness of stability it is seen that $\gamma_{\max }$ is in general smaller than the minimum of these bounds. That means that the upper bound (3.18), although quite close to $\gamma_{\max }$, is not sharp and gives optimistic results in general.

As a specific numerical example, for $\alpha=0$ the upper bound on robustness of stability computed as the infimum of (5.9) is 0.5774, while the overall bound of Glover and McFarlane is 0.5921. However $\operatorname{DDRS}(\gamma)$ is solvable if and only if $\gamma \leq \gamma_{\max }=0.5228$. Taking for instance $\gamma=0.5$, an $R H_{\infty}$ function $\Omega(s)$ satisfying the condition $\|\Gamma(s)\|_{\infty} \leq \gamma^{-1}$ is given by

$$
\Omega(s)=\frac{-s^{3}+1.214 s^{2}+17.5298 s+15.3158}{s^{3}+16.7579 s^{2}+29.4578 s+25.8017} .
$$

Eliminating linearly dependent columns of $\Gamma(s)$ gives an image representation of the subspace valued function $\Pi \mathcal{C}(s)$. A state space realization of the controller is computed from the image representation as

$$
\begin{aligned}
\dot{x}_{c}(t) & =\left[\begin{array}{cc}
-1.9079 & 7.1786 \\
2.4861 & -14.8499
\end{array}\right] x_{c}(t)+\left[\begin{array}{cc}
0 & 1.2920 \\
0 & -5.8619
\end{array}\right] y(t) \\
u(t) & =\left[\begin{array}{ll}
0 & 3.2365
\end{array}\right] x_{c}(t)+\left[\begin{array}{ll}
-1 & 0
\end{array}\right] y(t) .
\end{aligned}
$$

This controller satisfies the disturbance decoupling requirement and has robustness margin 0.5014 which is indeed larger than $\gamma=0.5$. It is worth noting that the controller is of dynamic state feedback form and a constant state feedback controller would have a smaller stability margin.

\section{Conclusion}

The disturbance decoupling problem with robust stability has been introduced as an extension of the classical disturbance decoupling problem to deal with the uncertainties in the plant description. By formulating the disturbance decoupling and robust stability requirements in terms of subspace valued functions, an upper bound on the achievable robustness of stability has been derived as a necessary condition for the solvability of the problem. Although this is the best possible bound that can be obtained by a finite dimensional geometrical analysis it has been shown that the actual robustness of stability is in general smaller and the pointwise geometrical analysis does not give sharp bounds. 


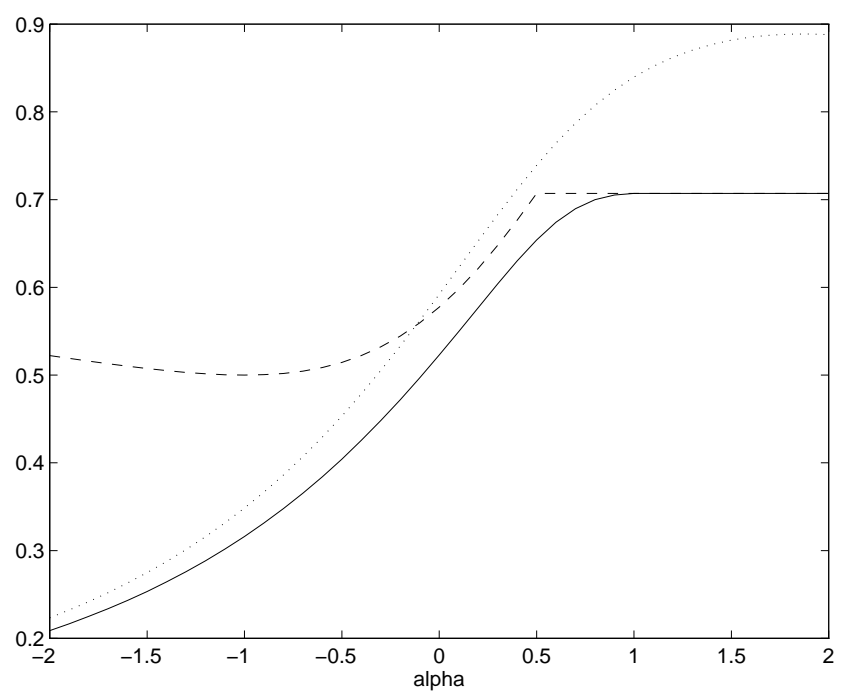

Figure 0.1: Constraint imposed by the disturbance decoupling requirement on robustness of stability. Dotted curve: Optimal overall bound of Glover and McFarlane. Dashed curve: Upper bound obtained by the pointwise geometrical analysis. Solid curve: Actually achievable robustness of stability.

For systems satisfying the left invertibility assumption 4.1, the set of all solutions of the disturbance decoupling problem with internal stability has been parametrized and the maximal achievable stability margin has been determined by optimizing over the free parameter. If assumption 4.1 does not hold the parametrization and the corresponding optimization problem become harder since there are more solutions. In forthcoming studies it is desirable to eliminate this assumption which essentially restricts the discussion to systems having equal number of control inputs and controlled outputs. It is also of interest to obtain a formula for the maximal stability margin that depends only on the plant parameters and not on the selection of a particular controller. Another interesting point could be to investigate the effect of the right half plane zeros of various subsystems on the solvability of DDRS.

\section{REFERENCES}

1. G. Basile And G. Marro (1992). Controlled and Conditioned Invariants in Linear System Theory. Prentice Hall, Englewood Cliffs, NJ.

2. M.K.K. Cevik and J.M. Schumacher (1995). The regulator problem with robust stability. Automatica 31, 1393-1406.

3. M.K.K. Cevik and J.M. Schumacher (1997). Regulation as an interpolation problem. Lin. Alg. Appl. 253, 311-340.

4. J. DE Does And J.M. Schumacher (1994). Continuity of singular perturbations in the gap topology. Lin. Alg. Appl. 205/206, 1121-1143.

5. B.A. Francis (1987). A Course in $H_{\infty}$ Control Theory. Lect. Notes Contr. Inf. Sci. 88. Springer, Berlin.

6. P.A. Fuhrmann (1981). Linear Systems and Operators in Hilbert Space. McGraw-Hill, New York.

7. T.T. Georgiou And M.C. Smith (1990). Optimal robustness in the gap metric. IEEE Trans. Automat. Contr. AC-35, 673-686.

8. K. Glover and D.C. McFarlane (1989). Robust stabilization of normalized coprime factor 
plant descriptions with $H_{\infty}$ bounded uncertainty. IEEE Trans. Automat. Contr. AC-34, 821-830.

9. I.C. Gohberg And M.G. Krein (1969). Introduction to the Theory of Linear Nonselfadjoint Operators. Transl. Math. Monogr. 18. Amer. Math. Soc., Providence, RI.

10. H. Imai AND H. AKashi (1981). Disturbance localization and pole shifting by dynamic compensation. IEEE Trans. Autom. Contr. AC-26, 226-235.

11. V. KUČERA (1974). Algebraic theory of discrete optimal control for multivariable systems. Kybernetika 10-21, 1-240, published in installments.

12. C. Martin and R. Hermann (1978). Applications of algebraic geometry to systems theory: The McMillan degree and Kronecker indices of transfer functions as topological and holomorphic system invariants. SIAM J. Contr. Optimiz. 16, 743-755.

13. A.B. ÖzGüler And V. Eldem (1985). Disturbance decoupling problems via dynamic output feedback. IEEE Trans. Autom. Contr. AC-30, 756-764.

14. L. QIU And E.J. Davison (1992). Pointwise gap metrics on transfer matrices. IEEE Trans. Automat. Contr. AC-37, 741-758.

15. M.G. Safonov, D.J.N. Limebeer, and R.Y. Chiang (1990). Simplifying the $H_{\infty}$ theory via loop shifting, matrix pencil and descriptor concepts. Int. J. Contr. 50, 2467-2488.

16. J. M. Schumacher (1980). Compensator synthesis using $(C, A, B)$ pairs. IEEE Trans. Autom. Contr. AC-25, 1113-1138.

17. J.M. Schumacher (1992). A pointwise criterion for controller robustness. Syst. Contr. Lett. 18, $1-8$.

18. M. Vidyasagar (1985). Control System Synthesis; A Factorization Approach. MIT Press, Cambridge, MA.

19. M. Vidyasagar And H. Kimura (1986). Robust controllers for uncertain linear multivariable systems. Automatica 22, 85-94.

20. J. C. Willems and C. Commault (1981). Disturbance decoupling by measurement feedback with stability or pole placement. SIAM J. Contr. Optim. 19, 409-504.

21. W.M. Wonham (1979). Linear Multivariable Control: a Geometric Approach, 2nd ed. Springer, New York.

22. D.C. Youla, J.J. Bongiorno And H.A. JabR (1976). Modern Wiener-Hopf design of optimal controllers. Part 2: The multivariable case. IEEE Trans. Automat. Contr. AC-21, 319-338.

23. K. Zhou with J.C. Doyle And K. Glover (1996). Robust and Optimal Control. Prentice-Hall, Upper Saddle River, NJ. 